\title{
MODELAGEM GEOTÉRMICA DA LITOSFERA NA REGIÃO ADJACENTE À BACIA SERGIPE-ALAGOAS, NE-BRASIL.
}

\author{
Das Flores, O. H. D. J' (DEG - UL, PPGEO - IGEO-UFBA) ; Dutra, A. C ${ }^{2}$ (DFTMA - IF -UFBA) \\ ${ }^{1}$ Departamento de Engenharia Geológica-Universidade Lúrio, Moçambique; Programa de pós-graduação em geofísica- \\ Instituto de Geociência-Universidade Federal da Bahia, Brasil. \\ ${ }^{2}$ Departamento de Física, Terra e Meio Ambiente, Instituto de Física, Universidade Federal da Bahia, Brasil.
}

Copyright 2021, SBGf - Sociedade Brasileira de Geofísica

This paper was prepared for presentation during the $17^{\text {th }}$ International Congress of the Brazilian Geophysical Society held in Rio de Janeiro, Brazil, 16-19 August 2021.

Contents of this paper were reviewed by the Technical Committee of the $17^{\text {th }}$ International Congress of the Brazilian Geophysical Society and do not necessarily represent any position of the SBGf, its officers or members. Electronic reproduction or storage of any part of this paper for commercial purposes without the written consent of the Brazilian Geophysical Society is prohibited.

Abstract

In this research we integrate geophysical and petrological data in order to build a geothermal model for the basement region adjacent to the Sergipe-Alagoas basin. The geophysical data used are bouguer, free-air and geoid anomalies, topography and heat flow data.

The method applied for modeling is based on the local isostasis. The first step of the work consisted on the filtering of geoid anomaly and topography data using lowpass in harmonic and frequency domains, respectively, to estimate the crustal and lithosphere thickness. The second step was characterized by geothermal modeling, were the crustal structure was restricted by geological information, thermophysical parameters, and results of gravimetric and magnetic data inversion, while the lithospheric mantle was restricted by chemical composition of xenolites.

The results show that maximum depths of moho and the thermal lithosphere ( $\mathrm{LAB}$ ) along to the modeled profile are $43 \mathrm{~km}$ and $194 \mathrm{~km}$ in alagoas sub-basin, which decreases along to the A-A' direction of the profile, and low temperatures are registed also in Alagoas sub-basin with an average of $587.3^{\circ} \mathrm{C}$ as moho temperature and 1343 ${ }^{\circ} \mathrm{C}$ in the $L A B$.

\section{Introdução}

Os estudos sobre a litosfera, sua dinâmica e a produção de calor na superfície terrestre vem ganhando destaque nos dias de hoje, principalmente para construir modelos geotérmicos que permitem fazer interpretação das características litosféricas atuais e também inferir sobre como ela responderia a diversas perturbações decorrentes do dinamismo tectônico, no espaço e no tempo, fornecendo assim a história geológica em uma região (Afonso et al., 2008).

A modelagem de dados geofísicos e petrológicos, associados a sua relação com o tectonismo de placas permitem determinar a estrutura e propriedades térmicas, anomalias de velocidade e distribuição de densidade na litosfera e topo da astenosfera.

Neste trabalho, apresentamos um estudo que integra dados de anomalias de gravidade (anomalias ar-livre e bouguer), anomalia de geóide, dados de topografia e fluxo de calor, condutividade térrmica e taxa de calor radiogênico, para construir um modelo geotérmico, de distribuição de densidade e de volocidade das ondas sísmicas ( $\mathrm{S}$ e $\mathrm{P}$ ) da litosfera nos diferentes domínios geotectônicos da região do da subprovincia Borborema Meridional, concretamente a bacia Sergipe-Alagoas e o terreno adjacente.

\section{Localização da área de pesquisa}

A bacia de Sergipe-Alagoas se localiza no nordeste brasileiro, nos estados de Sergipe e Alagoas. Este estudo foi realizado nos domínios geotectônicos resultados da colisão entre o maciço Pernambuco-Alagoas e o Cráton do São Francisco, e que constituem o embasamento adjacente à bacia de Sergipe-Alagoas (figura 1).

Esse embasamento adjacente compreende os domínios geotectônicos Salvador-Esplanada-Boquim (SEB) da sub-província do Cráton do São Francisco e os domínios Estância, Canudos-Vaza-Barris, Canindé, Macururé, Coruripe e Pernambuco-Alagoas, e fazem parte da 
Modelagem geotérmica da litosfera na região adjacente à bacia sergipe-alagoas, NE-Brasil.

sub-província Meridional da Província Borborema (Santos et al. 2000, Sampaio, 2019).

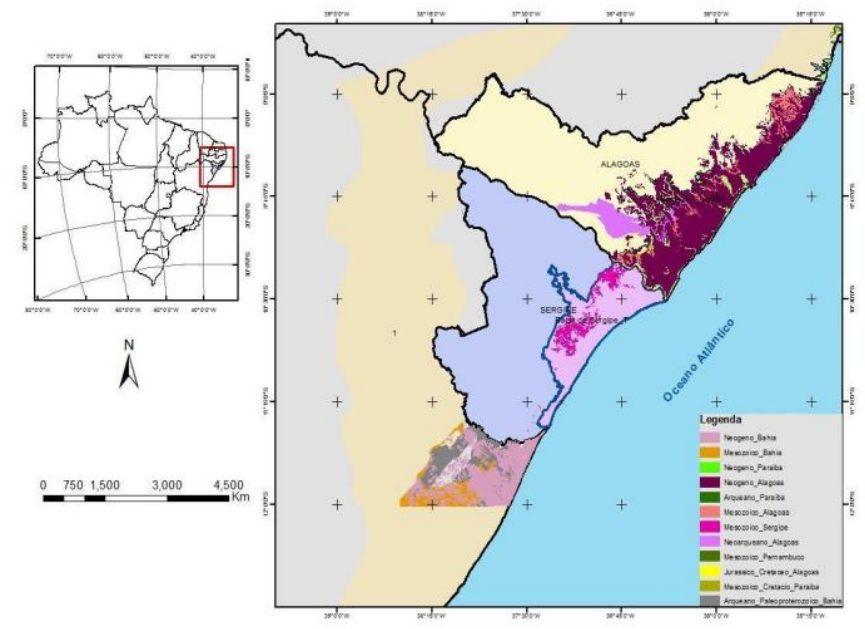

Figura 1: Mapa de localização da Bacia SergipeAlagoas.

\section{Método}

Para atingir o escopo deste trabalho, adotou-se como primeira etapa a obtenção de dados de gravidade (anomalias ar-livre e bouguer), elevação (topografia), anomalia de geóide e dados geotérmicos (fluxo de calor, condutividade térmica e taxa de calor radiogênico). $O$ fluxograma do processamento de dados é representado pela figura 2.

Os dados de topografia foram extraídos do ETOPO1, modelo de relevo global, disponível na página da Administração Oceânica e Atmosférica Nacional (http://www.ngdc.noaa.gov/mgg/global/global.html) esses dados foram interpolados e construiu-se o mapa da topografia (figura 3 ). Os dados de anomalia ar-livre (figura 4) foram extraídos do BGI (https://bgi.obs-mip.fr/), utilizando o modelo EGM 2008.

Os dados de anomalias bouguer (figura 5) e de geóide (figura 6) foram extraídos do ICGEM (http://icgem.gfzpotsdam.de) utilizando os modelos GECO (Gilardoni et al., 2016) e EIGEN-6C4 (Forste, Christtoph et al., 2014), respectivamente.

Esses dois modelos completos apresentam grau harmônico esférico e ordem igual 2190.



Figura 2: Fluxograma de processamento de dados.

Portanto, para evitar efeitos de variações densidade no manto sublitosférico, removeu-se assinaturas geoidais dos coeficientes harmônicos até grau e ordem 10, aplicando o filtro low-pass no domínio harmônico (Tunini et al., 2014), isto é, calculou-se a anomalia do geóide até o grau e ordem 10 que corresponde a anomalia regional e subtraíu-se ela da anomalia total para obter a anomalia residual (figura 6) ou seja: $N_{r e s}=N_{t}-N_{\text {reg }}$

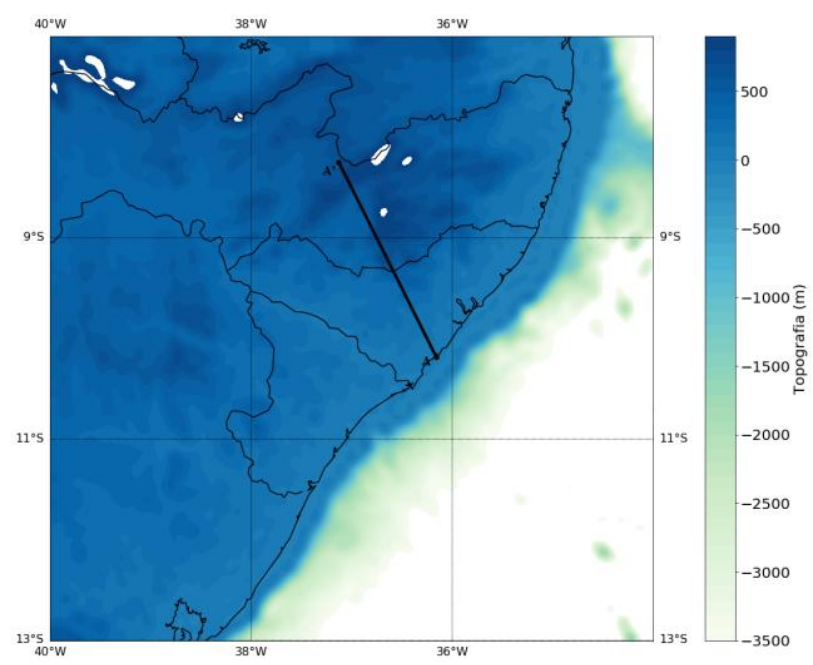

Figura 3: Mapa da elevação (topografia).

Onde: $N_{t}$ é a anomalia de geóide total; $N_{\text {reg }}$ é a anomalia do geóide regional; e $N_{\text {res }}$ é a anomalia de geóide residual. 


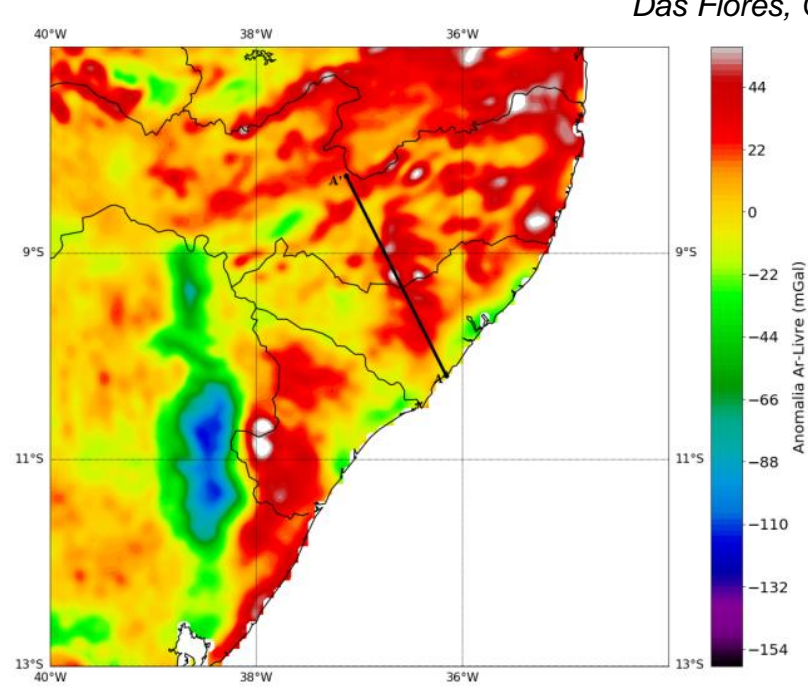

Figura 4: Mapa da anomalia Ar-Livre (em mGal).

Os dados geotérmicos (fluxo de calor, condutividade térmica e taxa de calor radiogênico) foram obtidos de campanhas terrestres do projeto Geoterm (IF-UFBA), da Rede Nacional de Gravimetria através do banco de dados da ANP (Petrobras e outras empresas petrolíferas) coadjuvado com informações da literatura. Na figura 7 , apresenta-se o mapa de fluxo de calor.

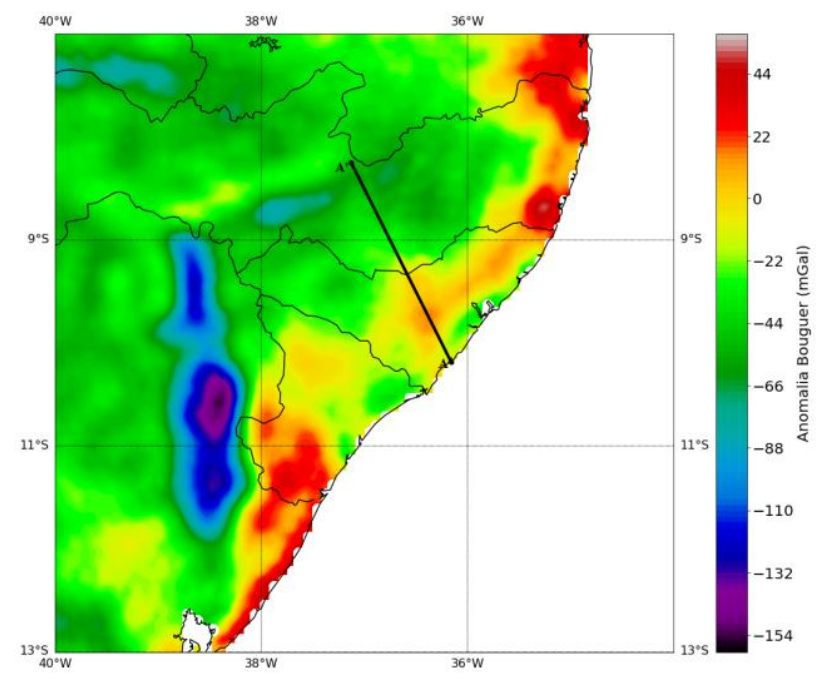

Figura 5: Mapa da anomalia bouguer (em mGal).

Para a modelagem utilizou-se o LitMod-2D (Afonso et al., 2008, Kumar et al, 2020), que é um código de elementos finitos baseado na integração de dados geofísicos e petrológicos para modelagem da litosfera num ambiente termodinâmico-geofísico consistente. Esses dados combinados permitem calcular a distribuição 2-D de temperatura, densidade e velocidade das ondas sísmicas. O domínio de modelagem é dividido por corpos que constituem a crosta, cada um com suas propriedades termofísicas previamente definidas e composição química para caso de corpos que compõem o manto.

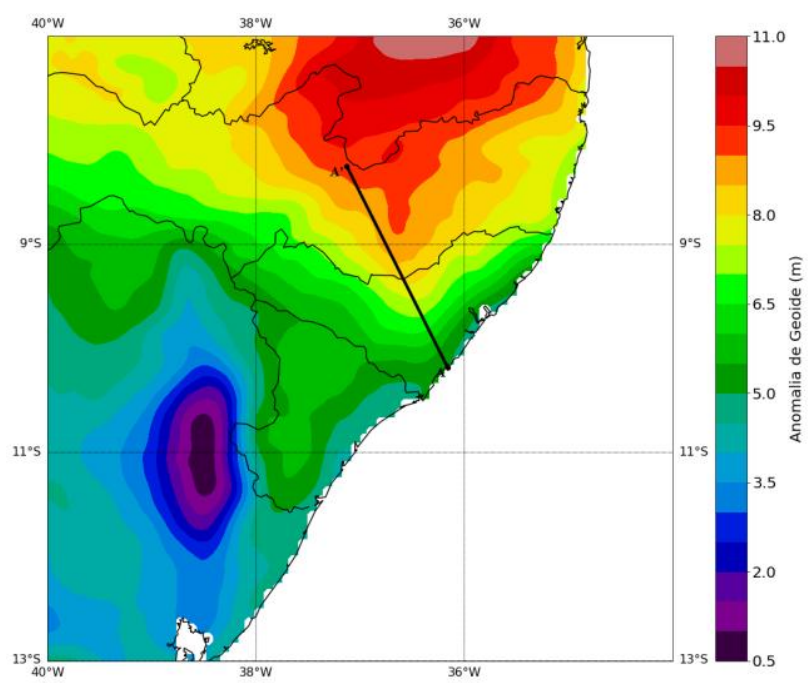

Figura 6: Mapa da anomalia de geóide filtrada (com coeficientes harmônicos até grau e ordem 10 removidos).

A distribuição de temperatura é calculda resolvendo a equação 2-D de transporte de calor em regime estacionário e considerando a taxa de calor radiogênico, dado por:

$$
\nabla \cdot(-\mathrm{k} \nabla \mathrm{T}-\mathrm{A}(\mathrm{x}, \mathrm{z})=0
$$

Onde: $k$ é a condutividade térmica e $A$ é a produção de calor radiogênico.

Com base na temperatura obtida na equação acima, determina-se a distribuição de densidade no manto litosférico, que é dependente da temperatura:

$$
\rho_{\mathrm{m}}=\rho_{\mathrm{a}}\left[1+\alpha\left(\mathrm{T}_{\mathrm{a}}-\mathrm{T}_{\mathrm{z}}\right)\right]
$$

Onde: $\rho_{\mathrm{a}}$ é a densidade da astenosfera, $\alpha$ é o coeficiente de expansão térmica e $\mathrm{T}_{\mathrm{a}}$ é a temperatura no limite litosfera-astenosfera.

Assumindo as seguintes condições de contorno: nenhuma perda de calor através nos limites laterais e temperaturas fixas na parte superior e inferior do modelo de 0 e $1320{ }^{\circ} \mathrm{C}$.

Portanto, a anomalia de gravidade (ar-livre e bouguer) é calculada usando o algoritmo 2-D de Talwani et al. 1959:

$$
\Delta g=2 G \rho \sum_{i=1}^{n} Z_{i}
$$


Modelagem geotérmica da litosfera na região adjacente à bacia sergipe-alagoas, NE-Brasil.

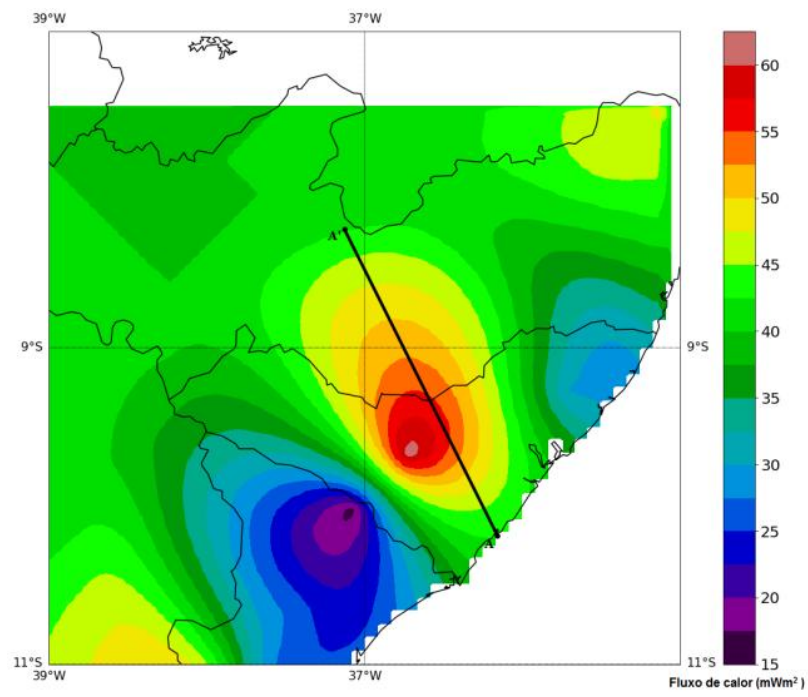

Figura 7: Mapa de Fluxo de Calor (em mWm²).

Onde: $\rho$ é a densidade, $G$ é a constante de gravitação universal e $Z_{i}$ é uma função relacionada aos vértices do corpos na superfície.

A anomalia do geóide $(\Delta N)$ é calculada usando um algorítmo de Nagy et al. 2000 e Zeyen et. al 2005, do potencial de gravidade para um prisma retangular com de densidade vertical:

$$
\Delta N=\frac{G \rho}{g} \int_{x_{1}}^{x_{2}} \int_{y_{1}}^{y_{2}} \int_{z_{1}}^{z_{2}} \frac{1}{\sqrt{x^{2}+y^{2}+z^{2}}} d z d y d x
$$

Onde: $g$ é a aceleração da gravidade terrestre; $x, y$ e $z$ são as coordenadas do prisma.

A topografia é calculada assumindo o equilíbrio isostático local com a profundidade de compensação dentro do domínio de modelagem.

Nas tabelas 1 e 2 estão os parâmetros físicos e composição química do manto litosférico utilizado na modelagem.

\begin{tabular}{|l|c|c|c|}
\hline \multicolumn{1}{|c|}{ Corpos } & $\rho\left(\mathrm{kg} / \mathrm{m}^{3}\right)$ & $K(W / K . \mathrm{m})$ & $A\left(\mu W / \mathrm{m}^{3}\right)$ \\
\hline sedimentos & 2650 & 2,5 & 2 \\
\hline Anfibolito & 2760 & 2,3 & 1,65 \\
\hline granito & 2720 & 2,4 & 1,67 \\
\hline Fonte rc & 2760 & 2,7 & 1,65 \\
\hline Fonte peal & 2660 & 2,4 & 1,65 \\
\hline Crosta média & 2800 & 2,1 & 0,5 \\
\hline Crosta inferior & 2950 & 2,0 & 0,2 \\
\hline
\end{tabular}

Tabela 1: Parâmetros físicos dos corpos utilizados na modelagem; $\rho$ é a densidade; $K$ é condutividade térmica e $A$ é a taxa de calor radiogênico.

\begin{tabular}{|c|c|c|c|}
\hline Ordem & Oxidos & Manto 1 & Manto 2 \\
\hline 1 & $\mathrm{SiO}_{2}$ & 43,12 & 44,5 \\
\hline 2 & $\mathrm{Al}_{2} \mathrm{O}_{3}$ & 4,25 & 3,5 \\
\hline 3 & $\mathrm{FeO}$ & 9,29 & 8,0 \\
\hline 4 & $\mathrm{MgO}$ & 37,76 & 39,78 \\
\hline 5 & $\mathrm{CaO}$ & 3,0 & 3,1 \\
\hline 6 & $\mathrm{Na}_{20}$ & 0,28 & 0.23 \\
\hline 7 & Total & 97,7 & 99,11 \\
\hline Tabela: Composição química do manto litosférico,
\end{tabular}

extraído do Ngonge et al., 2019 e Griffin et al., 2009.

\section{Resultados}

Após o processamento realizado no item anterior, seguiuse então para modelagem, que começou com o a integração de informações geológicas dos domínios da área de estudo, parâmetros físicos (densidade, condutividade e produçâo de calor radiogênico), profundidade e forma geométrica das fontes crustais, e composição química para o manto litosférico (tabela 2). A figura 8 , mostra o perfil $A-A^{\prime}$, do modelo crustal de melhor ajuste encontrado. Cada litologia é caraterizado pelo seu próprio parâmetro físico descrito na tabela 1.

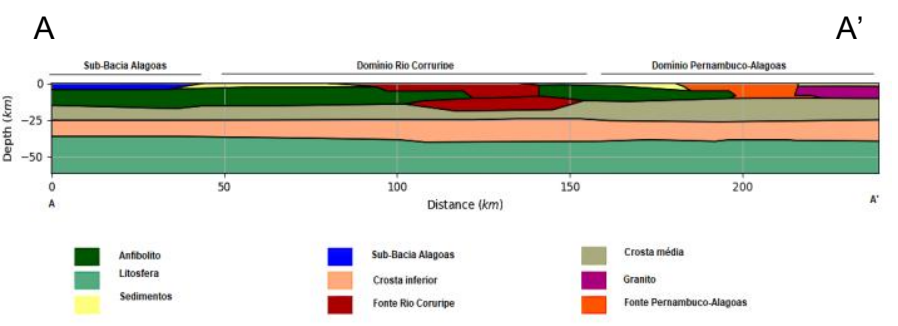

Figura 8: Modelo crustal de melhor Ajuste para o perfil $A-A^{\prime}$.

O embasamento cristalino é caraterizado pelas crostas média e inferior. Os corpos ao longo do perfil A-A' apresentam uma profundidade aproximadamente $17 \mathrm{~km}$ no domínio Rio Corruripe e $12 \mathrm{~km}$ para domínio Pernambuco-Alagoas, e $4 \mathrm{~km}$ de cobertura sedimentar 
Das Flores, O. H. D. J; Dutra, A. C

para sub-bacia Alagoas. Os sedimentos da são da era cenozoíca.

$\mathrm{Na}$ figura 9 são apresentados os resultados de dados de gravidade, geóide, topografia e fluxo de calor, do perfil AA' com o melhor ajuste, suas estruturas crustais foram descritos acima. O ajuste dos dados são mostrados na tabela 3 através cálculo do raiz do erro quadrático médio (RMSE) entre os dados observados e calculados, que foi determinado de acordo com o número total de pontos ao longo do perfil.



Figura 9: Modelo de melhor ajuste encontrado para o perfil A-A; a) Fluxo de calor; b) Anomalia de gravidade ar-livre; c) Anomalia de geóide; d) Anomalia de gravidade bouguer e e) Topografia.

\begin{tabular}{|c|c|c|c|c|}
\hline Perfil & $\begin{array}{c}\text { Anomalia } \\
\text { Bouguer } \\
\text { (mGal) }\end{array}$ & $\begin{array}{c}\text { Anomalia } \\
\text { Ar-livre } \\
\text { (mGal) }\end{array}$ & $\begin{array}{c}\text { Geóide } \\
\text { (m) }\end{array}$ & $\begin{array}{c}\text { Topografia } \\
\text { (m) }\end{array}$ \\
\hline RMSE & 4,604695 & 4,032032 & 0,08496 & 63,28399 \\
\hline
\end{tabular}

Tabela3: Resultado do cálculo do RMSE.

A composão química do manto litosférico desempenha um papel imprescendível no ajuste dos dados. A figura 10 , mostra os efeitos produzidos pela composicão do manto na densidade e na velocidade das ondas sísmicas, em uma litosfera com espessura de $194 \mathrm{~km}$ e profundidade da crosta (moho) de $43 \mathrm{~km}$.

A distribuição de temperatura é mostrado na figura 10a) e é caraterizada por isotermas horizontais com temperaturas dentro do manto litosférico. Na sub-bacia alagoas a temperatura Moho varia entre 585 à $603{ }^{\circ} \mathrm{C}$, enquanto que no manto litosférico a temperatura varia entre 1289 à $1343{ }^{\circ} \mathrm{C}$, nos domínios Rio Coruripe e Pernambuco-Alagoas a temperaturas de moho variam entre $605{ }^{\circ} \mathrm{C}$ à $632{ }^{\circ} \mathrm{C}$, enquanto que a temperatura no
LAB varia entre 1301 à $1320{ }^{\circ} \mathrm{C}$. Ao longo do perfil temperatura aumenta do domínio Pernambuco-Algoas à sub-bacia Algoas.

Como referido anteriormente, a distribuição de densidade no manto litosférico é dependente da temperaturapressão. No perfil, 10 b) mostra a distribuição de densidade no manto litosférico, onde se observa que baixas densidades $\left(3050 \mathrm{~kg} / \mathrm{m}^{3}\right)$ são registadas no domínio Rio Corruripe que está relacionada com o afinamento da manto litosférico nesse domínio, enquanto que altas densidades $\left(3200 \mathrm{~kg} / \mathrm{m}^{3}\right)$ são registradas no domínio Pernambuco-Alagoas.



Figura 10: Modelos do perfil A-A': a) Distibuição de temperatura; b) Distribuição de densidade no manto litosférico; c) Velocidade Sísmica das ondas $P$ e d) Velocidade Sísmica das ondas $P$.

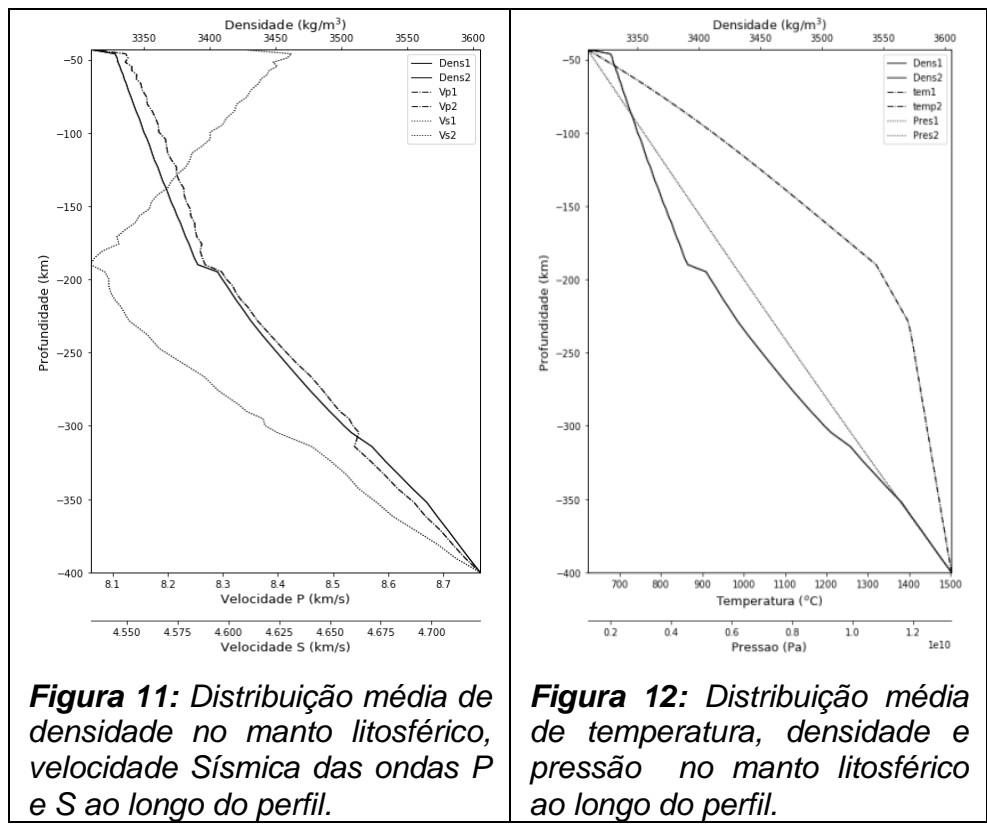


Modelagem geotérmica da litosfera na região adjacente à bacia sergipe-alagoas, NE-Brasil.

A figura $10 \mathrm{c}$ e d) mostram a anomalia da velocidade sísmicas para as ondas $\mathrm{P}$ e $\mathrm{S}$ ao longo do perfil. Observando essas figuras, constata-se que as velocidades das ondas $P$ aumentam com a profundidade dentro do manto litosférico e descem até $400 \mathrm{~km}$ de profundidade, enquanto as velocidades das ondas $\mathrm{S}$ diminuem com a profundidade até no $L A B$ e voltam aumentando novamente para a parte inferior do modelo, como se pode observar na figura 11. Na figura 12 apresenta-se o gráfico da distribuição média de temperatura, pressão e densidade em profundidade.

\section{Conclusão}

A integração de dados geofísicos e petrológicos permitiu construir um modelo de distribuição de temperatura e de densidade para a região do embasamento adjacente a bacia sergipe-Alagoas, além de determinar velocidade sísmicas para as ondas $\mathrm{P}$ e $\mathrm{S}$, anomalias de gravidade e de geóide, fluxo de calor e topografia.

Contudo, os resultados indicam que profundidades máximas de moho e da litosfera térmica ( $L A B)$ ao longo do perfil A-A' são de 43 km e 194 km observadas na subbacia, que vai diminuindo ao longo do perfil no domínio Rio Coruripe (40km de moho e 184-188 km para o LAB) e volta a aumentar no domínio Pernumbo-Alagoas (43 km de moho e $190 \mathrm{~km}$ para o LAB).

No tocante as temperaturas observa-se que baixas temperaturas são registradas na sub-bacia Alagoas com uma média de $587,3{ }^{\circ} \mathrm{C}$ na interface crosta-manto e $1343{ }^{\circ} \mathrm{C}$ no $\mathrm{LAB}$, ao passo que nos domínios Rio coruripe e Pernambuco-Alagoas vão 605 à $634{ }^{\circ} \mathrm{C}$ na interface crosta-manto e entre 1289 à $1320{ }^{\circ} \mathrm{C}$ para o LAB.

\section{Agradecimentos}

Agradecemos ao Promob - Cenpes - Petrobras pelo apoio através do projeto Petrobras 0050.0082543.13-9 e à CPRM, ANP e ICGEM pelos dados fornecidos e a todos os pesquisadores contribuintes deste projeto.

\section{References}

DUTRA, Alanna Costa. Determinação da espessura da interface sedimento-embasamento da Bacia Sergipe Alagoas a partir da assinatura gravimétrica. Revista de Geociências do Nordeste, v. 3, n. 1, p. 28-47, 2017.

Fullea, J., et al. "A rapid method to map the crustal and lithospheric thickness using elevation, geoid anomaly and thermal analysis. Application to the Gibraltar Arc System, Atlas Mountains and adjacent zones." Tectonophysics 430.1-4 (2007): 97-117.

Sampaio,Mariana Rosário Conceição Sampaio. Interpretação e modelagem de dados geofísicos no embasamento adjacente à bacia de Sergipe-Alagoas. Trabalho de graduação. Universidade Federal da Bahia.

Tunini, Lavinia, et al. "Geophysical- petrological model of the crust and upper mantle in the India- Eurasia collision zone." Tectonics 35.7 (2016): 1642-1669.

Jiménez- Munt, I., Torné, M., Fernandez, M., Vergés, J., Kumar, A., Carballo, A., \& García- Castellanos, D. (2019). Deep seated density anomalies across the Iberia- Africa plate boundary and its topographic response. Journal of Geophysical Research: Solid Earth, 124(12), 1331013332.

Griffin, W.L., O’Reilly, S.Y., Afonso, J.C. \& Begg, G., 2009. The composition and evolution of lithospheric mantle: a re-evaluation and its tectonic implications, J. Petrol., 50, 1185-1204.

Ngonge, E. D., de Hollanda, M. H. B. M., Puchtel, I. S., Walker, R. J., \& Archanjo, C. J. (2019). Characteristics of the lithospheric mantle beneath northeastern Borborema Province, Brazil: Re-Os and HSE constraints on peridotite xenoliths. Journal of South American Earth Sciences, 96, 102371. 